\title{
Incorporation of a laser range scanner into an image-guided surgical system
}

\author{
David M. Cash ${ }^{* a}$, Tuhin K. Sinha ${ }^{\mathrm{a}}$, William C. Chapman ${ }^{\mathrm{b}}$, Hiromi Terawaki ${ }^{\mathrm{c}}$, Michael I. Miga ${ }^{\mathrm{a}}$, \\ Robert L. Galloway, \\ ${ }^{a}$ Department of Biomedical Engineering, Vanderbilt University, Nashville, TN, USA 37235-1631; \\ ${ }^{b}$ Department of Surgery, Washington University School of Medicine, St. Louis, MO, USA 63110; \\ ${ }^{\mathrm{c}}$ Department of Biomedical Engineering, Duke University, Durham, NC, USA 27708; \\ ${ }^{\mathrm{d}}$ Department of Neurosurgery, Vanderbilt University, Nashville, TN, USA 37235;
}

\begin{abstract}
Laser range scanners provide rapid and accurate non-contact methods for acquiring three-dimensional surface data, offering many advantages over other techniques currently available during surgery. The range scanner was incorporated into our image-guided surgery system to augment registration and deformation compensation. A rigid body, embedded with infrared diodes, was attached to the scanner for tracking in physical space with an optical localization system. The relationship between the scanner's coordinate system and the tracked rigid body was determined using a calibration phantom. Tracking of the scanner using the calibration phantom resulted in an error of $1.4 \pm 0.8 \mathrm{~mm}(\mathrm{n}=234)$. Once tracked, data acquired intraoperatively from the range scanner data is registered with preoperative tomographic volumes using the Iterative Closest Point (ICP) algorithm. Sensitivity studies were performed to ensure that this algorithm effectively reached a global minimum. In cases where tissue deformation is significant, rigid registrations can lead to inaccuracy during surgical navigation. Methods of non-rigid compensation may be necessary, and an initial study using a linearly elastic finite element model is presented. Differences between intraoperative and preoperative surfaces after rigid registration are used to formulate boundary conditions, and the resulting displacement field deforms the preoperative image volume. To test this protocol, a phantom was built, consisting of fiducial points and a silicone liver model. Range scan and CT data were captured both before and after deforming the organ. The pre-deformed images, after registration and modeling, were compared to post-deformation CT images to evaluate this technique. The rigid registration accounts for most of the error from deformation, although there is a noticeable improvement by implementing the finite element model. To improve accuracy, more elaborate surface registration and deformation compensation strategies will be investigated. The range scanner is an innovative, uncumbersome, and relatively inexpensive method of collecting intraoperative data. It has been integrated into our image-guided surgical system and software with virtually no overhead.
\end{abstract}

Keywords: Laser range scanning, iterative closest point (ICP) algorithm, soft tisse deformation, image-guided surgery

\section{INTRODUCTION}

Hepatic metastases are the most common form of cancer in the liver found in the United States and other developed countries. These tumors often develop from primary colorectal cancer. Of the 148,300 estimated new cases of colorectal cancer that will occur in $2002^{1}$, it is estimated that $50 \%$ of all colorectal primary tumors will develop a liver metastasis at some point in the disease, and $20 \%$ of cases will develop a metastasis solely in the liver ${ }^{2}$. Metastatic liver cancer takes a very rapid course. If left untreated, the average life expectancy is approximately two months with fiveyear survival rate approaching zero.

The most common, and successful, method of treatment for metastatic liver tumors is surgical resection. For surgical resection, various studies have shown a five-year survival rate ranging from $22-60 \%$. Operative morbidity is a concern, with rates as high as $11 \%$. Another issue is subject eligibility; $70-90 \%$ of all patients with liver tumors are ineligible for surgical resection, due to overall health, lack of healthy liver, or the location of the tumor with respect to critical

* dave.cash@vanderbilt.edu; phone 1.615.343.8261; fax 1.615.343.7919; www.tgt.vanderbilt.edu and bmlweb.vuse.vanderbilt.edu 
structures ${ }^{3,4,5} 6$. Less invasive alternatives have been proposed, and include cryoablation, radiofrequency ablation, percutaneous ethanol injection, and chemoembolization. Complete removal of the tumor is very difficult when using these techniques, leading to the potential of recurrence. The effectiveness of these techniques is dependent on the ability to deliver therapy accurately to the target tumor while avoiding critical structures, such as the vasculature and biliary duct tree.

Image-guided surgery provides an accurate method of delivering therapy through the process of mapping the physical location of the anatomy to high-resolution pre-operative image volumes. The mapping provides the potential of realtime updating of surgical instrument position with respect to the image data. These concepts were initially developed for neurosurgical procedures, and there has been some initial work for application to hepatic surgery. Marescaux ${ }^{7}$ developed an extensive surgical planning suite, which implemented biomechanical modeling and haptic sensing for virtual surgery applications. Carrillo ${ }^{8,9}$ implemented manual and automatic registration techniques to guide an RF ablation probe using an interventional MR unit. Our group has performed registrations studies involving phantoms, animals, and humans using the liver surface ${ }^{10,11,12,13}$.

Three obstacles have been determined to be the most significant in the development of open-abdominal image-guided surgical procedures of the liver. First, there are no point-based landmarks which can be used as fiducials or targets in rigid registration methods. Once the procedure has begun, the liver is detached from the immobilizing ligaments so that better surgical access can be obtained. Thus, the liver moves independently and no other organ can be used for landmark data. In addition, landmarks that are easy to localize (e.g. portal vein bifurcation, suprahepatic vena cava, infrahepatic vena cava, tip of right lobe) may change position with respect to each other between preoperative imaging and intraoperative acquisition. The next issue is that there is significant movement of the liver due to respiration. Studies have shown this movement to be primarily cranial-caudal, and up to $3-4 \mathrm{~cm}^{14,15}$. The final challenge is tissue deformation. The shape of the liver has been determined to change from its preoperative state. The amount of shape change and how much it affects targeting accuracy needs to be determined, so that methods to account for it can be developed.

With these three considerations in mind, an image-guided surgery system for use with liver procedures has been developed. This system is based on the framework that has been used successfully for image-guided neurosurgical procedures at Vanderbilt and has two additional components. The first component is a means of acquiring threedimensional surface data through the use of a laser range scanner. This scanner outputs a dense set of three-dimensional point data that is used to register with preoperative images by means of a rigid surface-based registration algorithm. Laser range scanning has been used for registration in neurosurgical procedures using mutual information techniques which align the cortical vasculature ${ }^{16,17}$, as well as surface based techniques used to match cortical surface topology in phantoms ${ }^{18}$ and features on the human face ${ }^{19}$. Range scanning also provides a rapid method of acquisition, so that a large amount of data can be acquired in one short period of held respiration. Initial laser range scans performed during surgery have shown a good fit with preoperative CT volumes, although there is some deformation present. The scanner must be tracked in physical space so it can be linked with other localization systems. The second component involves implementing correction mechanisms for tissue deformation, based on the resulting range scan data. These corrections can be rooted in biomechanics through implementation of a finite element modeling technique, or they could be more interpolative in nature by introducing splines. The paper describes the details of this system, along with some illustrative cases from phantoms and surgery.

\subsection{Surgical Navigation Software}

\section{METHODS}

The Operating Room Image-Oriented Navigation (ORION) system was created at Vanderbilt University to handle the tasks required for an image-guided surgical procedure. ORION was developed under Windows NT/2000 using Microsoft Visual C++ 6.0 and Win 32 API. The two main objectives of ORION are to provide real-time updating of surgical position on medical images and to allow for flexibility and future functionality. Under the current framework, ORION is capable of rendering updates at $40 \mathrm{~Hz}$ on tomographic image sets. To provide flexibility, ORION's architecture consists of the main program that handles universal and integrative tasks during image-guided surgery and independent modules, which are designed to handle more specialized tasks and are loaded into the system as needed. 
Each module is implemented in the form of a DLL with an interface that is dependent on the type of task that it performs. Localizer DLLs handle the interface with physical space tracking systems. Currently, localizer DLL's exist for the OPTOTRAK 3020 and POLARIS (Northern Digital, Waterloo Ontario) optical localization systems, and for the FASTRAK(Polhemus, Colchester, VT) magnetic localization system. One for the AURORA (also Northern Digital) magnetic localization system is under development. Display DLLs handle the task of putting medical image data to the screen as well as displaying the surgical position in relationship to these images. This data could be in a conventional tomographic format or pre-rendered three-dimensional visualizations of the organ. The necessary link between localizer data and image data is performed using Registration DLLs. Modules have been written to perform point-based rigid registrations using Horn's quaternion method ${ }^{20}$, surface-based rigid registrations using the Iterative Closest Point algorithm $^{21}$, and projective 3D to 2D registrations using the Direct Linear Transform ${ }^{22}$. Graphic DLLs can be used to provide additional image information, including color-coded overlay for functional neurosurgery and live surface rendering. Finally, I/O DLL's handle the transfer of data from other modalities for intraoperative use. I/O DLLs have been implemented to display and acquire information captured from neurostimulators, video devices, and ultrasound data.

\subsection{Surface Acquisition}

To acquire dense surface information of the liver during surgery, a laser range scanner was used. The laser range scanners used in these experiments (RealScan200C, 3D Digital Corp., Bethel, CT) works on the optical principle of triangulation. Laser light is emitted from the range scanner and illuminates the surface. The light reflected from the surface is received by a CCD array within the range scanner, which has a known geometrical relationship with respect to the source. The depth is calculated based on this relationship and the calculated center of the received light pattern. The laser range scanner used in our surgical navigation system spreads the point source out into a vertical stripe to simultaneously calculate numerous range scan sample points. It is capable of scanning 500 lines per scene, and as many as 512 samples per line. In addition, when scanning at 256 samples per line, a video image is taken directly after range scan acquisition, and this image is texture mapped onto each point by a pre-determined calibration function, so that color information can be acquired in every point. While the color information is not used for this specific registration algorithm, it proves to be very useful in the identification of the liver within an intraoperative scene. Total acquisition time takes 5-20 seconds. An example of an intraoperative range scan is displayed in Figure 1.

In order for range scan data to be used in the same coordinate system as tracked surgical instruments, it must also be tracked by the optical localization device (OPTOTRAK 3020). A rigid body is affixed to the range scanner, and this rigid body contains Infrared Emitting Diodes (IREDs), which are recognized by the localizer. The relationship between the coordinate systems described by this rigid body and the range scanner is determined through a calibration procedure using a phantom. The phantom, shown in Figure 2, consists of nine separate platforms of varying height. In each platform, a white cylindrical disc of radius $9.5 \mathrm{~mm}$ was inserted flush to the platform. The rest of the platform was painted black so that most of the range scan data of the phantom not representing the disk was eliminated, thus making identification of each disk easier. From the range scan of the phantom, all nine discs were identified and their centroids were calculated. A hemispherical divot of $3 \mathrm{~mm}$ diameter was precisely machined in the center of each disk, so that when the $3 \mathrm{~mm}$ ball tip of a tracked surgical probe was placed into the divot, the centroid of the ball tip corresponded to the centroid of the disk. The resulting two sets of data could be used to determine a registration between range scan space and physical space. With this registration, and the position and orientation of the rigid body affixed to the range scanner, a calibration transformation is established. This transformation is between the range scanner and the affixed rigid body, and it will remain fixed throughout the procedure. A graphical representation of the calibration process Figure 2. 


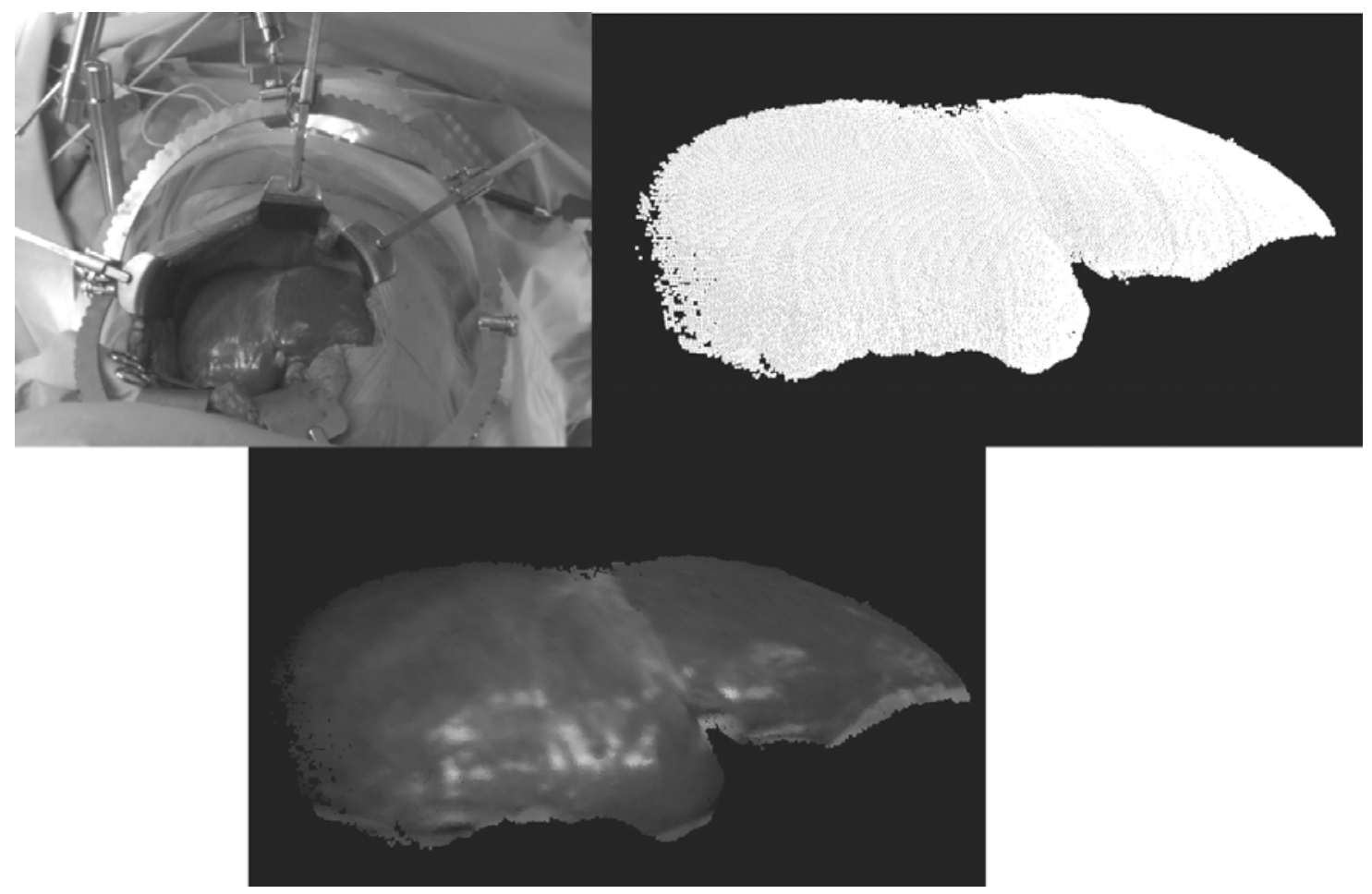

Figure 1 Laser range scan of an intraoperative scene. A video image is acquired of the scene by the laser scanner and texture mapped onto the range data (b), so that each point contains color information
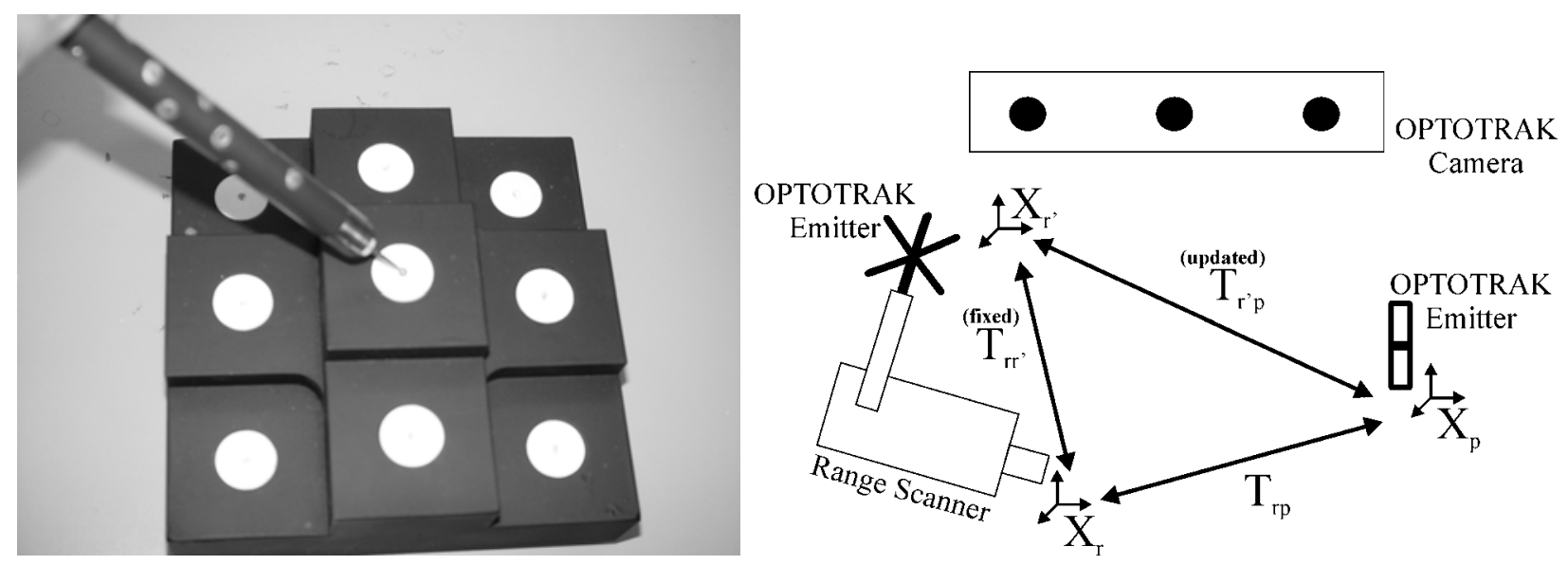

Figure 2 (Left) Calibration phantom, dimensions: $15.0 \mathrm{~cm}(\mathrm{~L}) \times 15.0 \mathrm{~cm}(\mathrm{~W}) \times 6.0 \mathrm{~cm}(\mathrm{H})$. Each disk is $9.5 \mathrm{~mm}$ in radius with a $3.0 \mathrm{~mm}$ diameter hemispherical divot machined in the center of each disk. Every platform is painted with non-reflective black paint to eliminate unwanted range scan data. (Right) Calibration diagram process, the calibration is used to determine the fixed transformation $\left(T_{r r}\right)$ between the range scanner $\left(X_{r}\right)$ and the OPTOTRAK emitter attached to it $\left(\mathrm{X}_{\mathrm{r}^{\prime}}\right)$. $\mathrm{T}_{\mathrm{rr}}$, is determined from the transformation between the range scanner and physical space $\left(\mathrm{T}_{\mathrm{rp}}\right)$, and from the OPTOTRAK, $\left(\mathrm{T}_{\mathrm{pr}}\right)$, which can be updated.

Two sets of experiments were performed regarding tracking of the range scanner. The first set of experiments involved registration experiments between the OPTOTRAK and range scanner. For every nine points of data generated in a trial, all 126 combinations of 5 targets and 4 fiducials were determined. A point-based registration for each of these sets was performed, and Fiducial and Target Registration Error were calculated. The second set of experiments that was 
performed involved using this phantom for tracking. Data was acquired from both physical space and range scanner space, and a calibration transformation was determined. This trial was deemed the calibration trial. Subsequent trials of data were then taken for both spaces. In between trials, the range scanner was brought out of and back into the field of view to simulate the process that will occur in surgery. Rather than registering the data, the calibration trial was used to determine the position of range scan points in physical space. These transformed points were used compared to physical data acquired by the range scan data. Every trial acquired was used one time as the calibration trial.

\subsection{Rigid Registration}

Rigid registration between image and physical data is achieved using the Iterative Closest Point algorithm ${ }^{21}$, modified to use k-d trees ${ }^{23,24}$ in order to decrease search time during closest point calculations. Abdominal CT volumes are acquired, and the liver is segmented from this images using manual delineation or a semi-automatic method based on the level-set method ${ }^{25}$. The contours were used in registrations with data from the range scanner and tracked probes. An initial alignment is required with the ICP algorithm in order to avoid local minimum, which is achieved using anatomical landmarks on the liver surface. To test the sensitivity of the surface based registration, a series of registrations were performed while perturbing the initial alignment by one of the degrees of freedom. These registrations were performed on one data set in which the inferior ridge was completely exposed, and another which only part of the inferior ridge was available.

While the Fiducial Registration Error is approximately $4-5 \mathrm{~mm}$ for landmark registration in phantoms, it is very difficult to localize landmarks during surgery. In clinical cases, Fiducial Registration Error based on landmarks can be as large as $25 \mathrm{~mm}$. To avoid relying on fiducial localization in the operating room, an initial alignment based on the Hotelling transform has been implemented. The Hotelling transform ${ }^{26}$ aligns the data sets according to their principal axes, which are calculated from the eigenvectors of the segmented point set's covariance matrix. Since only a fraction of the liver is exposed to the range scanner, a preprocessing step of eliminating non-exposed surface data from the image contours is necessary. In virtually all open abdominal procedures, a majority of the anterior surface of the liver as well as the inferior ridge at Sections III, IV, and V of the Couinaud segments ${ }^{27}$ is available for data acquisition. A reference direction is assigned in order to avoid right-handed coordinate systems in which two axes are inverted. This reference direction is assigned at the time of surface acquisition to determine the direction of two of the principal axes. A convention was decided that the reference direction would point caudally towards the left lobe, and that this would represent the positive $\mathrm{x}$ and $\mathrm{y}$ principal axes.

\subsection{Compensation of Soft Tissue Deformation}

Figure 3 shows a registration between image data and range scan data from a clinical case. From visual inspection, there is a noticeable shape change between the preoperative imaging studies and intraoperative presentation. Compensation for this deformation after rigid registration is being considered. In preliminary studies, a three-dimensional linearly elastic finite element model has been chosen for the compensation. The data is first registered using a rigid surface registration, and then modeling is performed using a finite element mesh generated from the preoperative image data. The resulting closest point correspondences at the end of the registration will be used to construct boundary conditions in terms of normal displacements. A more thorough description of this process, along with its application to clinical data, is presented in another paper at this conference ${ }^{28}$.

Deformation studies were performed using a liver phantom. The phantom consisted of three components: a poly (dimethyl) siloxane (rubber silicone) model of the liver, seven white Teflon spheres (Small Parts Inc. Miami, FL) machined to $12.70 \mathrm{~mm}$ with a $25 \mu \mathrm{m}$ tolerance, and a black Plexiglass base to eliminate unwanted range scan data. Fiducial data was acquired by fitting the range scan representation of each sphere's surface using a least-squares geometric method ${ }^{29}$. All of the components are easily differentiated in both range scan and CT images. The phantom was imaged with $\mathrm{CT}$, and a range scan was acquired immediately after, while keeping the phantom on the imaging gantry. This set of data served as a preoperative baseline. Then, a plastic cylindrical object of $3.7 \mathrm{~cm}$ height was placed underneath the phantom for the purpose of deformation. Another CT volume and range scan were acquired while the organ was deformed. Mock tumors made of Styrofoam spheres were placed in the organ while constructing the mold, and the centroid of these tumors in both preoperative non-deformed and intraoperative deformed volumes were identified. Distances between corresponding tumors were calculated after a point-based registration based on the Teflon 
spheres to determine the initial displacement, after a rigid surface registration using ICP, and after correction using the finite element model.
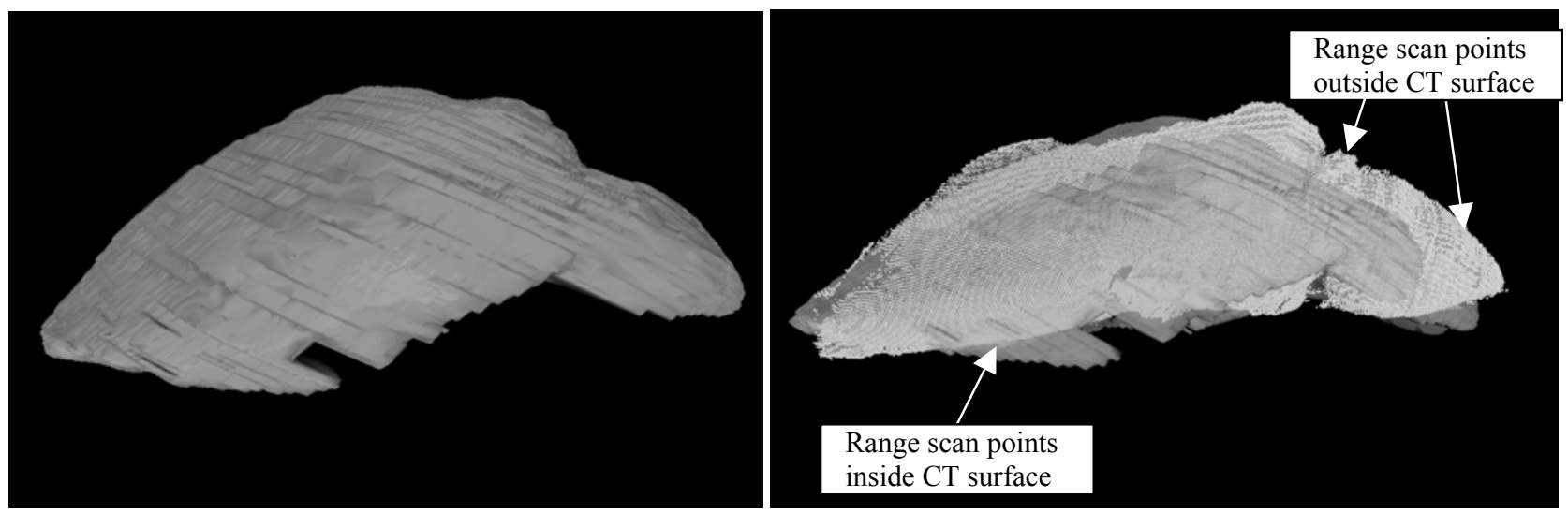

Figure 3 (Left) Triangulated surface from segmented CT data, (Right) Registered range scan point data overlaid on the surface. The white arrows point areas where deformation is exhibited.

\subsection{New components for ORION}

In order to handle the requirements of surface acquisition and deformation compensation during image-guided liver surgery, two new components were developed for ORION. The first component was an I/O DLL designed to communicate with the laser range scanner for data acquisition. The range scanner is connected to the surgery computer through a Universal Serial Bus (USB) connection, and the software interface developed by the manufacturer is written as an ActiveX control. The DLL was implemented in C++ using MFC. Once the data is acquired, it is displayed in one of the four display windows, where it could be rotated, zoomed, edited, and saved to disk. A screen shot from the range scanner acquisition is shown in Figure 4.

To handle compensation strategies, a new correction class of DLL was developed. This correction DLL handles nonlinear manipulation of images, such as finite element modeling and non-rigid registration. A description file, detailing the parameters and data to be used in the correction process is passed into ORION, and on to the correction DLL. For the finite element model, this file will hold the locations of the node, element, and boundary descriptions of the mesh to be used. The DLL will generate and warp the preoperative image, such that a new image is formed which has the same rigid registration as the preoperative image. Corrections will be performed as needed during surgery, using intraoperative data from ultrasound and range scanning.

\subsection{Clinical acquisition}

Data was acquired using the ORION surgical guidance system at two sites: Vanderbilt University Medical Center in Nashville, TN and Washington University Medical Center/Barnes-Jewish Hospital at Saint Louis, MO. During patient preparation, the OPTOTRAK camera, range scanner, and computer were brought into the operating room. Surface points are acquired using a tracked surgical probe in contact with the liver surface. The probe acquires new points at a rate of $40 \mathrm{~Hz}$, or a total of 2400 points per minute. During acquisition, the patient undergoes a brief anesthetically induced apneic period. During this apneic period, $100 \%$ Oxygen is placed into the airways so that the blood stream receives enough $\mathrm{O}_{2}$ through diffusion. However, there is a concern about $\mathrm{CO}_{2}$ buildup during the apneic periods, so $\mathrm{pH}$ is carefully monitored by the anesthesiologist. Range scan surfaces of the operative scene are acquired, as are regions of the liver by placing a tracked probe directly on the surface. Most of the tracked probe data will be for specific areas of interest, such as the inferior ridge or the falciform groove. 


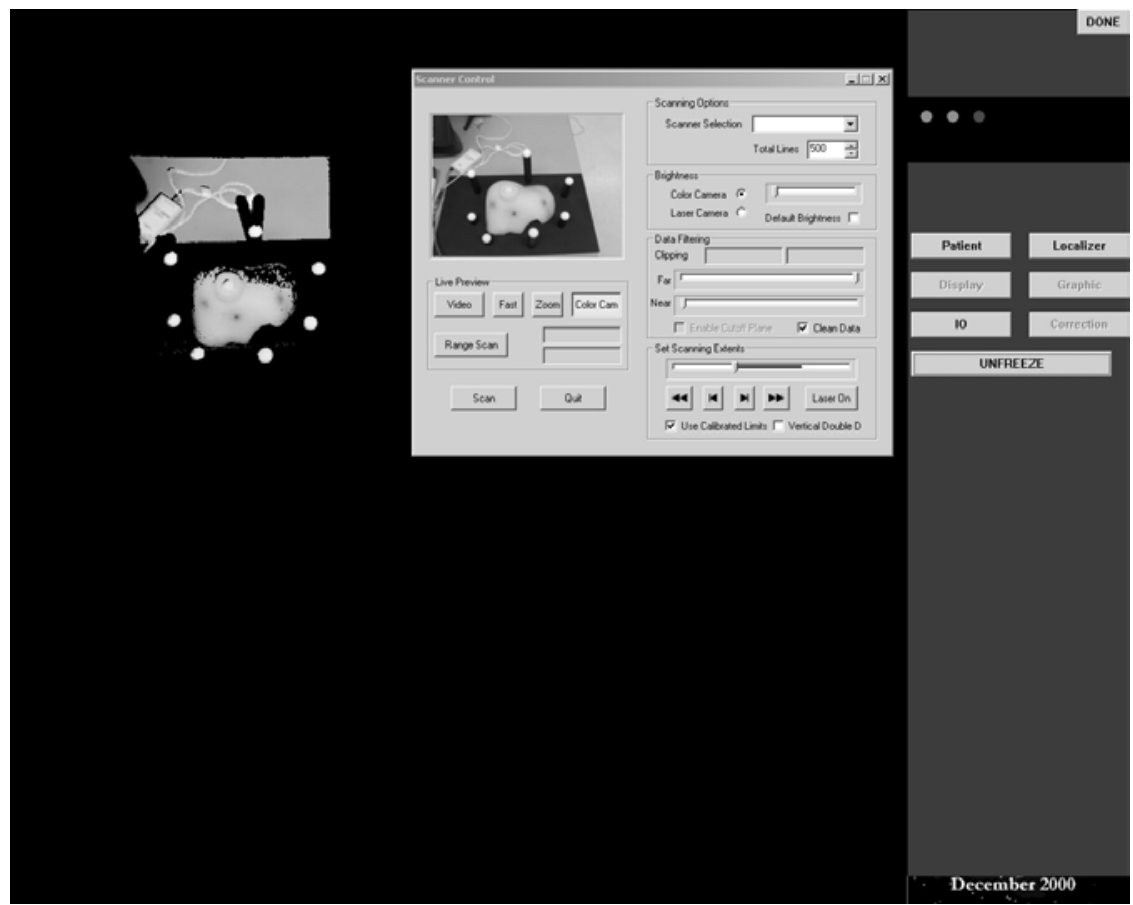

Figure 4 Screenshot of rangescan module in ORION surgical navigation software.

For range scanning in the OR, the tracking calibration procedure is performed before the patient is placed in the operating room. The range scanner is mounted on a surgical arm, so that it can easily be moved out of the surgeon's way. The scanner is brought near to the surgical field over the lower abdomen, and acquires a scan 2-3 feet away from the exposed liver. Preparation for a scan takes about 60 seconds, in which holding the patient's respiration is not necessary. Then, there is an apneic period during a 20 second range scan, and afterwards, surgery resumes while image processing and registration take place.

\subsection{Tracking results}

\section{RESULTS}

A total of twelve registration trials were performed using the calibration phantom. From the nine points available for each trial, there were a total of 126 unique combinations of 5 fiducials and 4 targets, which resulted in a total of 7,560 individual fiducial error measurements and 6048 individual target error measurements. The mean fiducial registration error was $1.02 \pm 0.56 \mathrm{~mm}$, with a maximum error $3.79 \mathrm{~mm}$, and the mean target registration error was $1.39 \pm 0.71 \mathrm{~mm}$, with a maximum of $4.81 \mathrm{~mm}$.

Tracking experiments were performed in two sets of trials, with five trials in one set and three trials in the other. Each trial served one time as the calibration trial, and using the calibration matrix from this trial, range scan points were transformed into physical space and compared to the actual OPTOTRAK configurations. This resulted in 36 samples for each trial in the first set ( 9 data points each for the four non-calibration trials) and 18 points for each trial in the second set. The overall tracking error with the phantom was $1.43 \pm 0.55 \mathrm{~mm}$ over 243 samples. The results from the tracking experiments are in Table 1. Figure 5 shows the application of the calibration procedure to the liver phantom. This figure shows the overlay of range scan data on top of physical space data of the liver phantom acquired with a tracked probe. No registration is performed between these two surfaces; they are aligned only by use of the calibration matrix and the position of the rigid body emitter affixed to the range scanner. The mean closest point distance for the overlapping regions of these surfaces is $1.5 \pm 1.0 \mathrm{~mm}(\mathrm{n}=2372$ points) for the left figure and $2.2 \pm 1.6$ ( $\mathrm{n}=2268$ points) for the right. 


\begin{tabular}{|c|c|c|}
\hline $\begin{array}{c}\text { Calibration } \\
\text { Trial }\end{array}$ & Samples & Tracking error, mm \\
\hline 1 & 36 & $1.25 \pm 0.51(2.35)$ \\
\hline 2 & 36 & $0.95 \pm 0.45(2.27)$ \\
\hline 3 & 36 & $1.58 \pm 0.97(4.34)$ \\
\hline 4 & 36 & $1.01 \pm 0.59(2.51)$ \\
\hline 5 & 36 & $2.12 \pm 0.95(3.68)$ \\
\hline 6 & 18 & $1.74 \pm 0.62(2.62)$ \\
\hline 7 & 18 & $1.46 \pm 0.60(2.32)$ \\
\hline 8 & 18 & $1.42 \pm 0.55(2.15)$ \\
\hline OVERALL & 234 & $1.43 \pm 0.79(4.34)$ \\
\hline
\end{tabular}

Table 1 Tracking results from the calibration phantom. Each trial served once as the calibration trial, and it's calibration matrix was used on all the other related sets of data.
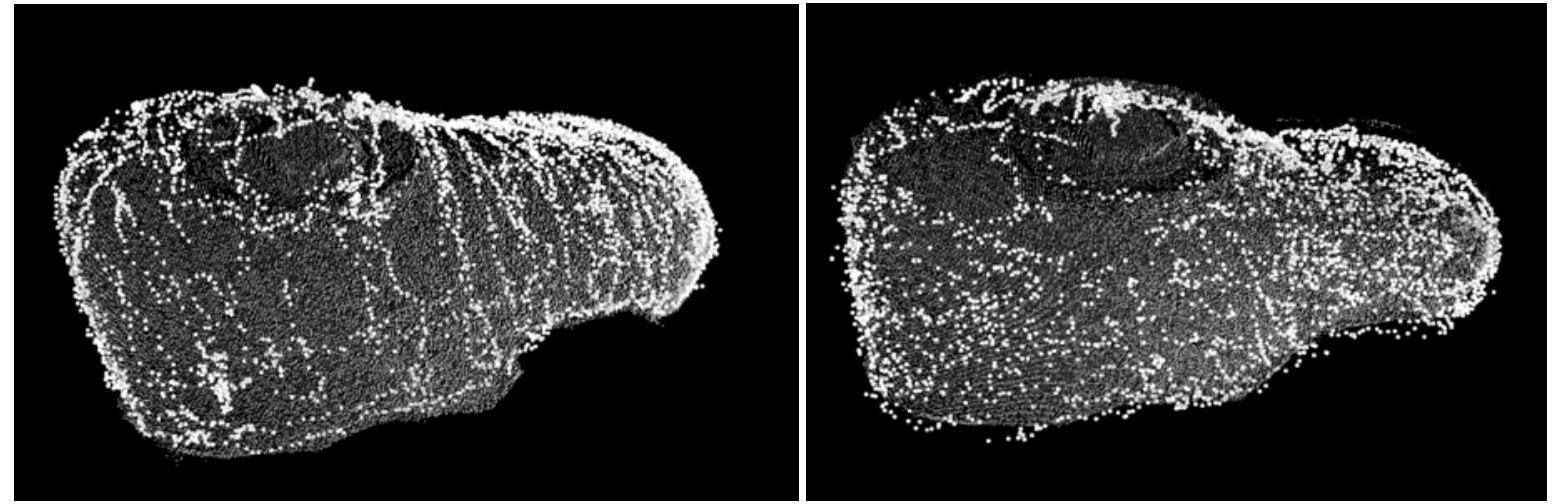

Figure 5 Results from range scanner tracking studies using the liver phantom. The light points represent physical space data acquired with a tracked surgical probe, while the dark points represent range scan data that has been transformed into physical space using the calibration procedure described in Sec. 2.3.

\subsection{Rigid registration}

Table 2 shows the results from the first sensitivity study. A successful range scan is defined based on visual alignment and mean closest point residual. Typically there is a difference on the order of 4-5 $\mathrm{mm}$ mean closest point residual between a "success" and a "failure". Of the 396 studies performed on data with an incomplete ridge, 30 failed, most occurring due to a rotation in the $\mathrm{X}$ and Z-axes. However, with a better definition of the ridge, only one trial failed out of 455. All the successful registrations were used to transform the physical data into image space, and a mean transformed position was calculated for each point in the physical data. A deviation from the mean points was calculated using all the successful registrations in order to determine the precision of the final results from each ICP registration.

\begin{tabular}{|c|c|c|c|c|c|c|}
\hline Ridge & Initial FRE & Trials & Failures & $\begin{array}{l}\text { Translation } \\
\text { range, mm }\end{array}$ & $\begin{array}{c}\text { Successful } \\
\text { Rotation range, } \\
\text { degrees }\end{array}$ & $\begin{array}{l}\text { Average distance } \\
\text { from mean success } \\
\text { position, } \mathrm{mm}\end{array}$ \\
\hline Incomplete & 16.2 & 396 & 30 & $\begin{array}{l}\text { X: }(-40,+40) \\
Y:(-40,+36) \\
Z:(-40,+40)\end{array}$ & $\begin{array}{l}X:(-25,+14) \\
Y:(-25,+25) \\
Z:(-10,+25)\end{array}$ & $0.24 \pm 0.06$ \\
\hline Complete & 19.1 & 455 & 1 & $\begin{array}{l}\text { X: }(-50,+50) \\
Y:(-50,+50) \\
Z:(-50,+50)\end{array}$ & $\begin{array}{l}\text { X: }(-25,+24) \\
Y:(-25,+25) \\
Z:(-25,+25)\end{array}$ & $1.3 \pm 0.2$ \\
\hline
\end{tabular}

Table 2 Sensitivity registration studies. The initial alignment was perturbed by one component and then registered using ICP. The ranges listed are those where a successful registration was reached. 
An additional sensitivity study was also performed to determine the robustness of the initial alignment by Hotelling transform. 100 trials were performed in which the initial transformation, either landmark-based or Hotelling-based, was perturbed randomly in all six degrees of freedom. The limit of perturbation was $(-20 \mathrm{~mm},+20 \mathrm{~mm})$ for all translations and $\left(-10^{\circ}, 10^{\circ}\right)$ for all rotations. In both cases, all 100 trials were successes, and the average distance from the mean registered position was $1.1 \pm 0.2 \mathrm{~mm}$ for the Hotelling transform and $0.9 \pm 0.1 \mathrm{~mm}$ for the landmark based transform.

\subsection{Deformation compensation}

The position of the six mock tumors in relation to the non-deformed surface is shown in Figure 6(a), and the difference of the CT surface before and after deformation is shown in Figure 6(b). Table 3 shows that the overall error between tumors decreased nearly $70 \%$ due to the rigid registration, although there was an increase in error for two of the tumors. Deformation compensation accounted for another $6 \%$ decrease in overall error, and no significant increases in error.
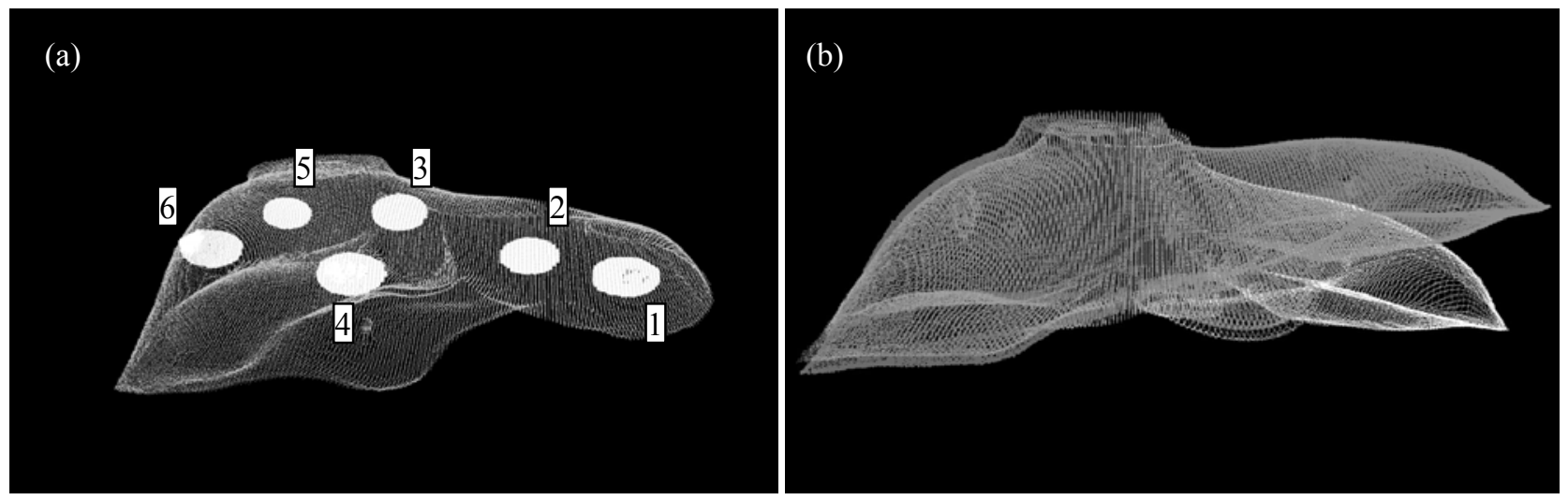

Figure 6 (a) Non-deformed liver surface from segmented CD surface. The white spheres indicate the location of the mock tumors, and the numbers coincide with Table 3. (b) CT surfaces from non-deformed and deformed image volumes. The plastic object was placed on the right side of the image.

\begin{tabular}{|c|c|c|c|c|c|c|}
\hline Tumor & $\begin{array}{c}\text { Initial error, } \\
\mathrm{mm}\end{array}$ & $\begin{array}{c}\text { Rigid Error, } \\
\mathrm{mm}\end{array}$ & $\%$ change & $\begin{array}{c}\text { Rigid + Deformation } \\
\text { error, } \mathrm{mm}\end{array}$ & $\begin{array}{c}\% \text { change } \\
\text { from initial }\end{array}$ & $\begin{array}{c}\text { \% change from } \\
\text { rigid }\end{array}$ \\
\hline 1 & 46.8 & 3.8 & $-91.8 \%$ & 1.9 & -4.1 & -50.0 \\
\hline 2 & 33.7 & 3.7 & $-89.0 \%$ & 2.4 & -3.9 & -35.1 \\
\hline 3 & 11.5 & 6.4 & $-44.0 \%$ & 5.0 & -13.0 & -23.2 \\
\hline 4 & 6.0 & 6.2 & $4.1 \%$ & 4.4 & -29.8 & -28.7 \\
\hline 5 & 3.7 & 6.6 & $80.7 \%$ & 6.5 & -4.4 & -2.4 \\
\hline 6 & 4.7 & 7.7 & $65.7 \%$ & 7.8 & 2.0 & 1.2 \\
\hline MEAN & 17.7 & 5.8 & -67.2 & 4.7 & -6.2 & -18.9 \\
\hline
\end{tabular}

Table 3 Results from the deformation studies. The first percentage reports the change between the initial error and the rigid error. The second percentage is the percent change between modeling and surface registration in terms of the initial error, while the last column indicates the amount of improvement with respect to the rigid error.

\subsection{Registration Targeting}

\section{DISCUSSION}

One of the primary challenges to this research is the validation of registration accuracy. Since there is no landmark data available for fiducials, there are also no landmarks that can serve as targets in order to verify the registration. However, there are regions on the liver that contain geometrically rich features and could be localized with enough confidence to serve in a manner similar to a point-based target. The two most likely candidates are the inferior ridge of the liver and the groove underneath the falciform ligament, which are highlighted in Figure 7. There have been many different uses of surface geometry for registration, including surface normals, principal curvatures, extremal points, crest and ridge lines (for a review of these techniques, see ${ }^{30}$ ). Furushiro et al. ${ }^{31}$ used ridgeline descriptions in a manner similar to 
fiducials for registration of a liver phantom. The results from Section 3.2 of this paper indicate that the inferior ridgeline could play a stabilizing role in the registration. This ridgeline provides more uniqueness to the surface being registered and eliminates some local minima. However, not all of the ridgeline may be needed for use in the registration. Initial studies have focused on using only part of the ridge data for the registration, allowing the rest of the ridge to be used as target data. Rather than calculating closest point distance for the target metric, the surface is tessellated, and normals are projected from each range scan point onto the image surface. While this still does not provide a one to one correspondence, it does allow the surface invariant features of the liver surface to play a greater role in the error calculation. Automatic detection of regions with unique geometric signatures and more advanced error metrics are being pursued. Once sufficient targeting on the surface has been achieved, more invasive targeting methods will be explored.

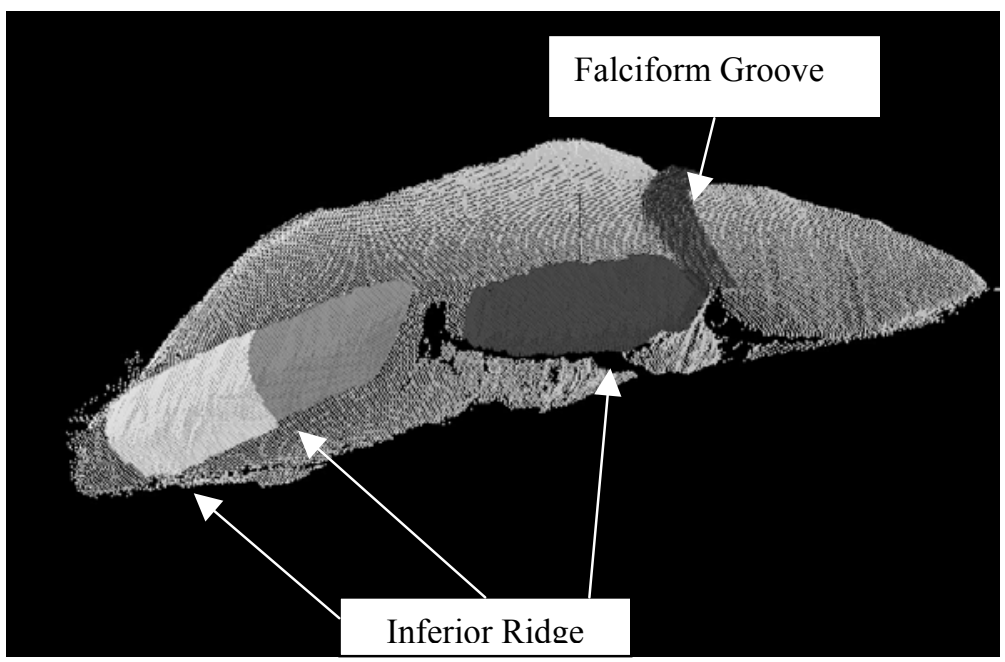

Figure 7 Target surfaces on the liver

\subsection{Deformation compensation strategy}

Finite element modeling has been widely examined in with respect to the problem of brain shift ${ }^{32,33,34,35,36}$. It provides an inexpensive way of updating during surgery compared to intraoperative tomographic imaging. In terms of the deformations experienced in brain shift, the example presented with the phantom in this paper may seem a bit extreme. However, the type of deformation that is experienced in open abdominal surgery is much more of a gross shape change than a subtle deformation. The rigid ICP-based registration reduces a considerable amount of error at areas of large deformation by spreading this error across the entire surface. Although this strategy is shown to be beneficial in the results presented here, the method does not provide information that characterizes the extent of deformation during intraoperative presentation of the liver. However, observations during surgery have indicated that certain regions of the liver apparently undergo less shape change. If these areas could be identified systematically a priori, modifications to the ICP algorithm could be employed (e.g. added regional weighting) to aid in differentiating deformation from rigid body motion. Other methods of deformation, such as spline-based interpolation will be compared with the finite element to determine which method is better suited to handle the non-rigid component of the shape change.

The deformation did show improvement in locating tumor centroids. However, the improvement could be larger in clinical cases, since the deformation experiment performed with the phantom does not completely represent the data acquired in surgery. First, there is significantly less coverage of the liver surface by the range scanner in the operating room. While about $52 \%$ of the entire surface area is acquired on the phantom, only $25 \%$ is available during surgery. In addition, the liver phantom has more geometrically unique regions available for the range scanner than is observed during surgery. Finally, there will be more than one source of deformation in the operating room, removing some of the rigid component of this deformation. These three factors result in a rigid registration that can provide better compensation for the deformation, thus leaving little room for improvement by the finite element model. 


\section{CONCLUSIONS}

The components and protocols for an image-guided liver surgery system were developed. The image-guided surgical system now includes integrated interfaces for rapid surface acquisition with a range scanner and non-rigid correction with a finite element modeling. Initial studies with phantom show that tracking is highly accurate, and that deformation correction can improve the error of subsurface targets; however for very large deformations, a rigid component that can be compensated through registration is more dominant. Various deformation strategies will need to be compared to determine which method will be optimal. Sensitivity studies on clinical data show that surface registrations are highly robust to changes in initial alignment if a well-defined ridge is present in both sets of data.

\section{ACKNOWLEDGEMENTS}

This work was supported in part by the National Institute of Health R21 Grant No. CA 91352-01. The authors would like to acknowledge Tina Herron, Nita Collins, and the rest of the CT staff in Vanderbilt University's Department of Radiology for their aid in acquiring tomographic images of the phantom and patients. In addition, the authors would like to thank Stephanie Cook and Karin Mayes of the Vanderbilt University Hepatic Surgery Nursing Team for their aid and patience with regards in acquiring intraoperative data. Assistance in constructing the phantoms was provided by John Fellenstein and Robert Patchin from the Department of Physics, and Phil Davis from the School of Engineering. Some segmentation and calculation were performed using Analyze AVW Version 3.1 - Biomedical Resource, provided in collaboration with the Mayo Foundation, Rochester, MN. Finally, the authors would like to acknowledge 3D Digital Corporation for their technical support and training regarding their laser range scanner.

\section{REFERENCES}

1. American Cancer Society, Cancer Facts and Figures-2002, www.cancer.org, 2002.

2. G. D. Steele, Jr., "The National Cancer Data Base report on colorectal cancer", Cancer, 74, 1979-1989, 1994.

3. A. Sardi, A. Akbarov, and G. Conaway, "Management of primary and metastatic tumors to the liver", Oncology (Huntington NY), 10, 911-925, 1996.

4. A. R. Sasson and E. R. Sigurdson, "Surgical treatment of liver metastases", Semin.Oncol., 29, 107-118, 2002.

5. $\quad$ P. Moroz, P. R. Salama, and B. N. Gray, "Resecting large numbers of hepatic colorectal metastases", ANZ.J.Surg., 72, 5-10, 2002.

6. C. K. Leow, W. Y. Lau, and A. K. Li, "Surgical resection of colorectal hepatic metastases", Hong.Kong.Med.J., 3, 50-56, 1997.

7. J. Marescaux, J. M. Clement, V. Tassetti and others, "Virtual reality applied to hepatic surgery simulation: the next revolution", Ann.Surg., 228, 627-634, 1998.

8. A. Carrillo, J. L. Duerk, J. S. Lewin and others, "Semiautomatic 3-D image registration as applied to interventional MRI liver cancer treatment", Ieee Transactions on Medical Imaging, 19, 175-185, 2000.

9. D. L. Wilson, A. Carrillo, L. Zheng and others, "Evaluation of 3D image registration as applied to MR-guided thermal treatment of liver cancer", Jmri-Journal of Magnetic Resonance Imaging, 8, 77-84, 1998.

10. A. J. Herline, J. D. Stefansic, J. Debelak and others, "Technical advances toward interactive image-guided laparoscopic surgery", Surgical Endoscopy, 14, 675-679, 2000.

11. A. J. Herline, J. L. Herring, J. D. Stefansic and others, "Surface registration for use in interactive, image-guided liver surgery", Comput.Aided Surg., 5, 11-17, 2000.

12. A. J. Herline, J. D. Stefansic, J. P. Debelak and others, "Image-guided surgery: preliminary feasibility studies of frameless stereotactic liver surgery", Archives of Surgery, 134, 644-649, 1999.

13. D. M. Cash, T. K. Sinha, W. C. Chapman, R. L. Galloway, and M. I. Miga, "Fast, accurate surface acquisition using a laser range scanner for image-guided liver surgery", SPIE Medical Imaging 2002, S. Mun, ed., 4681, 2002.

14. S. Shimizu, B. Shirato, B. Xo and others, "Three-dimensional movement of a liver tumor detected by highspeed magnetic resonance imaging", Radiotherapy and Oncology, 50, 367-370, 1999.

15. H. W. Korin, R. L. Ehman, S. J. Riederer and others, "RESPIRATORY KINEMATICS OF THE UPPER ABDOMINAL ORGANS - A QUANTITATIVE STUDY", Magnetic Resonance in Medicine, 23, 172-178, 1992. 
16. T. K. Sinha, D. M. Cash, R. J. Weil and others, "Cortical Surface Registration Using Texture Mapped Point Clouds and Mutual Information", Medical Image Computing and Computer-Assisted Intervention - Miccai'02, 2489, 533-540, 2002.

17. M. I. Miga, T. K. Sinha, D. M. Cash and others, "Cortical Surface Registration for Image-Registration for Image-Guided Neurosurgery Using Laser-Range Scanning", IEEE Trans.Med.Imaging, In Press, 2003.

18. M. A. Audette, K. Siddiqi, and T. M. Peters, "Level-set surface segmentation and fast cortical range image tracking for computing intrasurgical deformations", Medical Image Computing and Computer-Assisted Intervention, Miccai'99, Proceedings, 1679, 788-797, 1999.

19. A. Raabe, R. Krishnan, R. Wolff and others, "Laser surface scanning for patient registration in intracranial image-guided surgery", Neurosurgery, 50, 797-801, 2002.

20. Horn B.K.P., "Closed-form solution of absolute orientation using unit quaternions", J.Opt.Soc.Amer., 4, 629642, 1987.

21. P. J. BESL and N. D. MCKAY, "A METHOD FOR REGISTRATION OF 3-D SHAPES", Ieee Transactions on Pattern Analysis and Machine Intelligence, 14, 239-256, 1992.

22. J. D. Stefansic, A. J. Herline, Y. Shyr and others, "Registration of physical space to laparoscopic image space for use in minimally invasive hepatic surgery", IEEE Trans.Med.Imaging, 19, 1012-1023, 2000.

23. J. H. Friedman, Bentley J.L., and Finkel R.A., "An Algorithm for Finding Best Matches in Logarithmic Expected Time", ACM Trans.Math Software, 3, 209-226, 1977.

24. Z. Y. ZHANG, "ITERATIVE POINT MATCHING FOR REGISTRATION OF FREE-FORM CURVES AND SURFACES", International Journal of Computer Vision, 13, 119-152, 1994.

25. S. Pan and B. M. Dawant, "Automatic segmentation of the liver from abdominal CT images: a level-set approach", SPIE Medical Imaging 2001, 4322, 2001.

26. H. Hotelling, "Analysis of a complex of statistical variables into principal components", J.Educ.Psychol., 24, 417-441, 1933.

27. C Couinaud, Le Foie: Etudes Anatomiques et Chirurgicales, Masson, Paris, 1957.

28. M. I. Miga, D. M. Cash, Z. Cao and others, "Intraoperative Registration of the Liver for Image-Guided Surgery Using Laser Range Scanning and Deformable Models", SPIE Medical Imaging 2003, 5029, 2003.

29. S. J. Ahn, W. Rauh, and H. J. Warnecke, "Least-squares orthogonal distances fitting of circle, sphere, ellipse, hyperbola, and parabola", Pattern Recognition, 34, 2283-2303, 2001.

30. M. A. Audette, F. P. Ferrie, and T. M. Peters, "An algorithmic overview of surface registration techniques for medical imaging", Med.Image Anal., 4, 201-217, 2000.

31. N. Furushiro, T. Saito, Y. Masutani and others, "Specification Method of Surface Measurement for Surgical Navigation: Ridgeline Based Organ Registration", Medical Image Computing and Computer-Assisted Intervention - Miccai'02, 2489, 109-115, 2002.

32. K. D. Paulsen, M. I. Miga, F. E. Kennedy and others, "A computational model for tracking subsurface tissue deformation during stereotactic neurosurgery", IEEE Trans.Biomed.Eng, 46, 213-225, 1999.

33. P. J. Edwards, D. L. G. Hill, J. A. Little and others, "Deformation for image guided interventions using a three component tissue model", Med.Image Anal., 2, 355-367, 1998.

34. O. M. Skrinjar and J. S. Duncan, "Real time 3D brain shift compensation", Information Processing in Medical Imaging, Proceedings, 1613, 42-55, 1999.

35. A. Hagemann, K. Rohr, H. S. Stiehl and others, "Biomechanical modeling of the human head for physically based, nonrigid image registration", IEEE Trans.Med.Imaging, 18, 875-884, 1999.

36. K. Miller and K. Chinzei, "Constitutive modelling of brain tissue: experiment and theory", Journal of Biomechanics, 30, 1115-1121, 1997. 